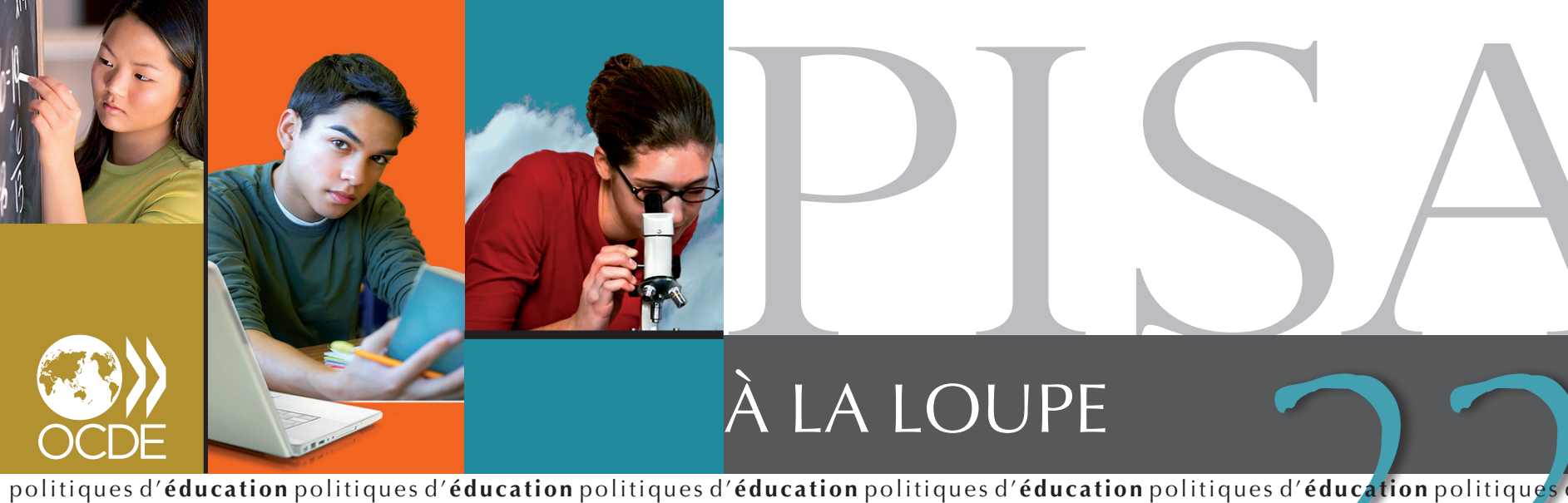

\title{
Où en sont les élèves issus de l'immigration dans les établissements d'enseignement défavorisés?
}

- Les élèves issus de l'immigration doivent souvent surmonter de nombreux obstacles à la fois pour réussir à l'école.

- Dans la plupart des pays de l'OCDE, la moindre performance des élèves issus de l'immigration par rapport aux autres êlèves est fortement liée au profil socio-économique défavorisé de leur établissement d'enseignement, caractérisé par la concentration d'élèves dont la mère est peu instruite.

- La concentration, dans un établissement d'enseignement, d'élèves issus de l'immigration ou d'élèves qui ne parlent pas la langue d'instruction à la maison ne présente pas une corrélation aussi forte avec une moindre performance scolaire.

Les politiques et pratiques choisies pour l'intégration des élèves issus de l'immigration dans le système d'éducation - et au sein de la société - font l'objet de vives réactions et de débats controversés. La forte concentration d'élèves issus de l'immigration ne parlant pas la langue d'instruction à la maison affecte-t-elle la performance scolaire de ces mêmes élèves et de leurs camarades de classe ? Quelle corrélation existe-t-il entre les écarts de performance entre élèves issus de l'immigration et élèves autochtones, d'une part, et les différences de profil socio-économique de l'effectif de l'établissement $d^{\prime}$ enseignement qu'ils fréquentent, d'autre part?

Les élèves issus de l'immigration sont confrontés à de nombreux défis en matière d’apprentissage..
Il existe un lien étroit entre désavantage socio-économique et statut d'immigrant. La plupart des immigrants quittent leur pays en quête de perspectives économiques meilleures. Une fois arrivés dans leur pays d'accueil, ils s'installent souvent dans des communautés regroupant d'autres immigrants partageant leur culture, leur langue et bien souvent, leur profil socio-économique. Les immigrants occupent souvent des emplois faiblement rémunérés et leurs possibilités d'emprunt sont limitées; le choix de logements à leur disposition est donc souvent plus restreint. Leurs enfants fréquentent souvent les mêmes établissements d'enseignement - et ces établissements présentent en général une forte concentration d'élèves issus de I'immigration. La répartition des élèves issus de l'immigration entre les établissements n'est, par conséquent, pas équitable ; en fait, ils tendent à se concentrer dans certains établissements d'enseignement. La plupart du temps, ces établissements d'enseignement présentent une concentration relativement importante d'élèves issus de l'immigration et un profil socio-économique plus défavorisé que les autres établissements. 
Démêler les défis multiples auxquels les élèves issus de l'immigration sont confrontés

Proportion de l'effectif total d'élèves issus de l'immigration qui fréquentent le quart d'établissements $d$ 'enseignement présentant la plus forte concentration...
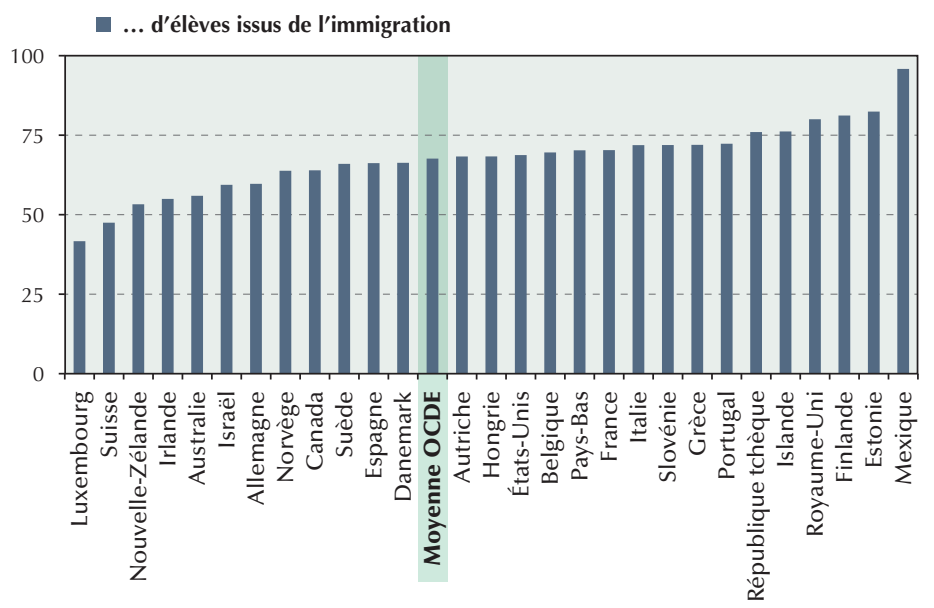

-... d'élèves issus de l'immigration

qui ne parlent pas la langue $d^{\prime}$ instruction à la maison

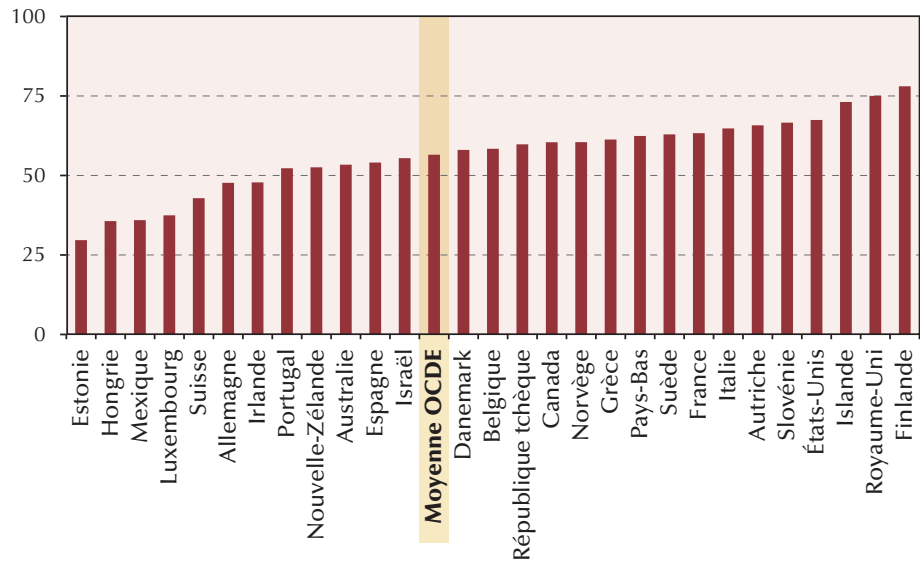

-... d'élèves dont la mère est peu instruite

(n'est pas titulaire d'un diplôme de fin d'études secondaires)

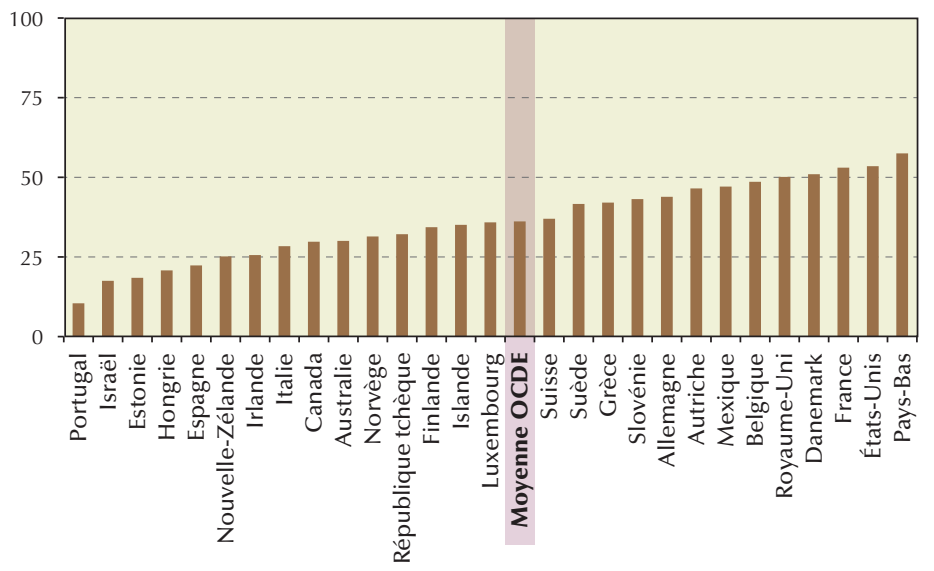

Remarque : Les pays sont classés par ordre croissant pour chaque indicateur de concentration. Source : OCDE (2012), Untapped Skills: Realising the Potential of Immigrant Students, figure 5.8 et tableau 5.4.
Ainsi, les élèves issus de l'immigration doivent souvent surmonter plusieurs obstacles à la fois pour réussir à l'école : la barrière linguistique, leur propre statut d'immigrant, un profil socio-économique défavorisé, et le fait que nombre de leurs camarades de classe sont confrontés aux mêmes difficultés qu'eux.

Démêler la relation entre le statut d'immigrant, les compétences linguistiques et le profil socio-économique n'est pas chose aisée. Les données de l'enquête PISA montrent toutefois comment ces trois variables sont liées entre elles et dans quelle mesure elles sont corrélées à la performance des élèves à l'école.

\section{... notamment le profil}

socio-économique de l'établissement d'enseignement où ils sont scolarisés.

Une analyse des données de I'enquête PISA 2009 s'est penchée plus particulièrement sur trois types d'établissements d'enseignement : ceux présentant la plus forte concentration d'élèves issus de l'immigration ; ceux présentant la plus forte concentration d'élèves issus de l'immigration qui parlent à la maison une autre langue que la langue d'instruction à l'école; et ceux présentant la plus forte concentration d'élèves dont la mère est peu instruite. Le fait d'avoir une mère peu instruite, c'est-à-dire qui n'est pas titulaire d'un diplôme de fin d'études secondaires, est un indicateur de désavantage socio-économique particulièrement pertinent au sein des populations issues de l'immigration.

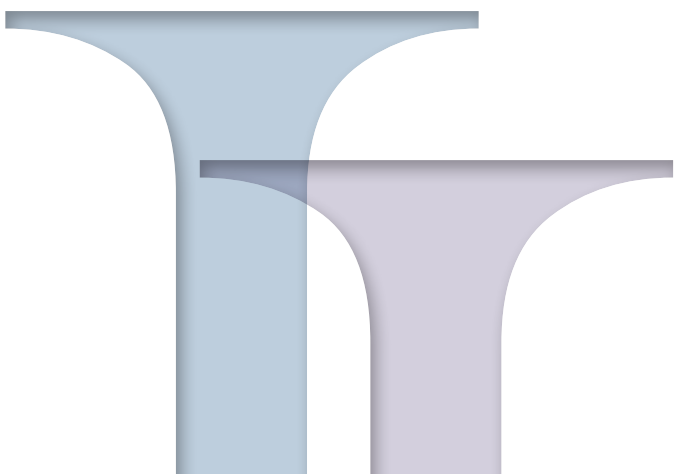



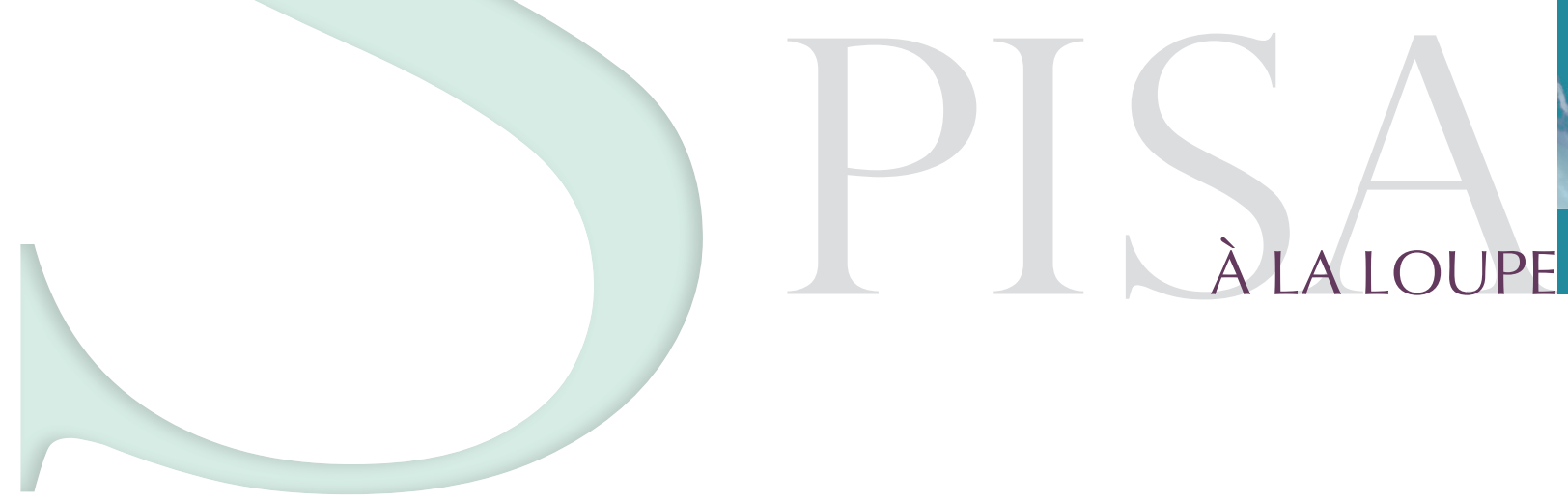

Les modalités d'accueil des élèves issus de I'immigration dans les établissements d'enseignement varient fortement selon les pays. En Nouvelle-Zélande, par exemple, $50 \%$ des élèves issus de l'immigration (soit bien en-deçà de la moyenne de l'OCDE de 68 \%) sont scolarisés dans un établissement d'enseignement présentant une forte concentration d'élèves issus de I'immigration. En outre, la concentration d'élèves issus de l'immigration dans les établissements d'enseignement présentant un profil socio-économique défavorisé est également relativement faible en Nouvelle-Zélande : seul un élève issu de l'immigration sur quatre (contre $36 \%$, en moyenne, dans les pays de I'OCDE) y fréquente un établissement d'enseignement présentant une forte concentration d'élèves dont la mère est peu instruite. En Allemagne, la concentration d'élèves issus de l'immigration dans les établissements d'enseignement est modérée (aux alentours de la moyenne de l'OCDE), tandis que la concentration d'élèves issus de l'immigration dans des établissements d'enseignement présentant un profil socio-économique défavorisé est supérieure à la moyenne de l'OCDE. Au Royaume-Uni, la forte concentration d'élèves issus de l'immigration dans les établissements d'enseignement s'accompagne d'une forte concentration d'élèves issus de l'immigration dans les établissements d'enseignement les plus défavorisés.

En analysant la performance des élèves au travers de ce prisme, il apparaît que c'est avec la concentration dans les établissements d'enseignement d'élèves dont la mère est peu instruite que la moindre performance scolaire des élèves, notamment de ceux issus de l'immigration, présente la plus forte corrélation. Ce constat laisse penser que, pour les élèves issus de l'immigration - comme pour tous les élèves, d'ailleurs -, le fait d'être concentrés dans des établissements d'enseignement fréquentés par des élèves confrontés au même désavantage socio-économique qu'eux constitue un obstacle majeur à leur réussite scolaire.

\section{La forte concentration d'élèves au profil socio-économique défavorisé dans un établissement d'enseignement est fortement corrélée à une moindre performance en compréhension de l'écrit parmi les élèves issus de l'immigration}

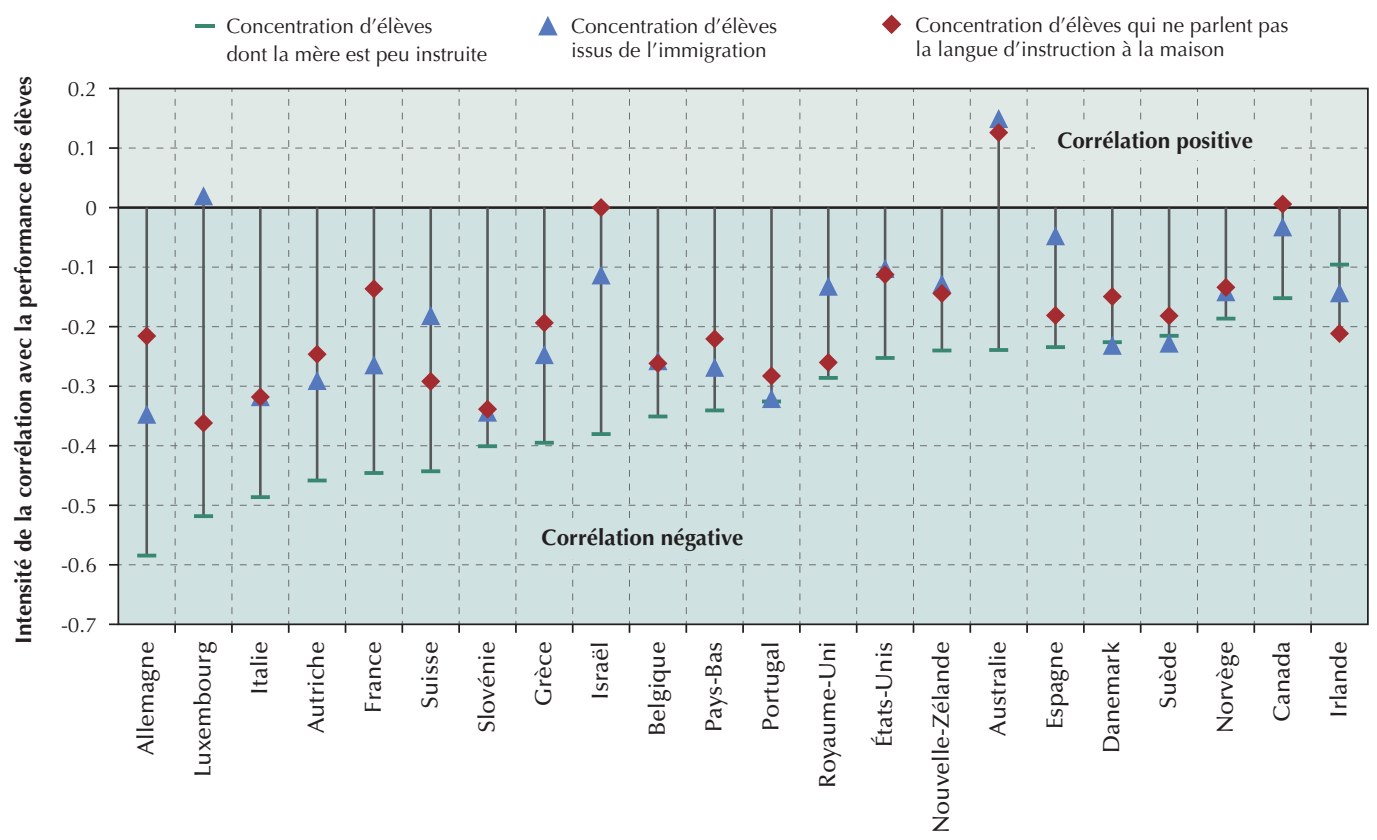

Remarque : Les pays sont classés par ordre croissant de l'intensité de la corrélation entre la performance des élèves et la concentration d'élèves dont la mère est peu instruite dans chaque établissement d'enseignement.

Source : OCDE (2012), Untapped Skills: Realising the Potential of Immigrant Students, figure 5.9 et tableau 5.6

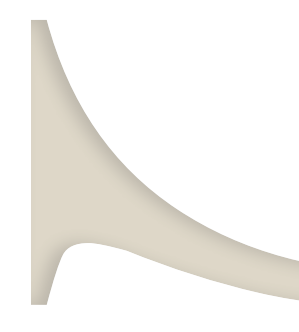


En revanche, les résultats suggèrent que la moindre performance des élèves n'est fortement corrélée ni avec la concentration d'élèves issus de l'immigration, ni avec celle d'élèves parlant une autre langue que la langue d'instruction. En d'autres termes, la scolarisation dans un établissement d'enseignement fréquenté par des élèves originaires d'autres pays ou parlant plusieurs langues n'affecte pas autant l'apprentissage que la fréquentation d'un établissement d'enseignement présentant une forte concentration d'élèves issus de milieux défavorisés, c'est-à-dire d'élèves dont la mère est peu instruite. Il existe en fait de nombreux établissements d'enseignement très performants qui présentent une forte concentration d'élèves issus de l'immigration. Leurs bons résultats résultent souvent de la mise en œuvre de politiques d'éducation nationales ou régionales conçues spécifiquement pour faciliter
I'accueil - et l'optimisation du potentiel d'effectifs d'élèves hétérogènes. Au Canada, de nombreux établissements d'enseignement de la province d'Alberta présentent ainsi ce profil. À titre d'exemple, les politiques d'immigration qui ciblent les individus très instruits peuvent également permettre de renforcer le niveau de performance des établissements d'enseignement. Dans d'autres cas, certains établissements d'enseignement privé très performants peuvent sélectionner spécifiquement des élèves venant $d$ 'autres pays en leur proposant des cursus reconnus au niveau international.

Pour conclure: La concentration d'élèves issus de l'immigration dans un établissement d'enseignement ou une classe ne constitue pas nécessairement un obstacle à la bonne performance des élèves. En revanche, la concentration d'élèves au profil socio-économique défavorisé est, quant à elle, fortement corrélée à l'obtention de moins bons résultats scolaires pour les élèves. La réduction de la concentration d'élèves au profil socio-économique défavorisé au niveau de chaque établissement d'enseignement constitue donc un bon préalable pour faciliter la réussite de l'intégration des élèves issus de l'immigration à l'école et, à terme, au sein de la société.

Pour tout complément d'information

Contacter Pablo Zoido (Pablo.Zoido@oecd.org)

Consulter OCDE (2012), Untapped Skills: Realising the Potential of Immigrant Students, Éditions OCDE et l'ensemble des tableaux y afférents.

Voir

www.pisa.oecd.org www.oecd.org/pisa/infocus
Prochain numéro

À quoi les élèves aspirent-ils à la fin de leurs études secondaires? 